\title{
On the Fractal Dimension of Isosurfaces
}

\author{
Marc Khoury and Rephael Wenger
}
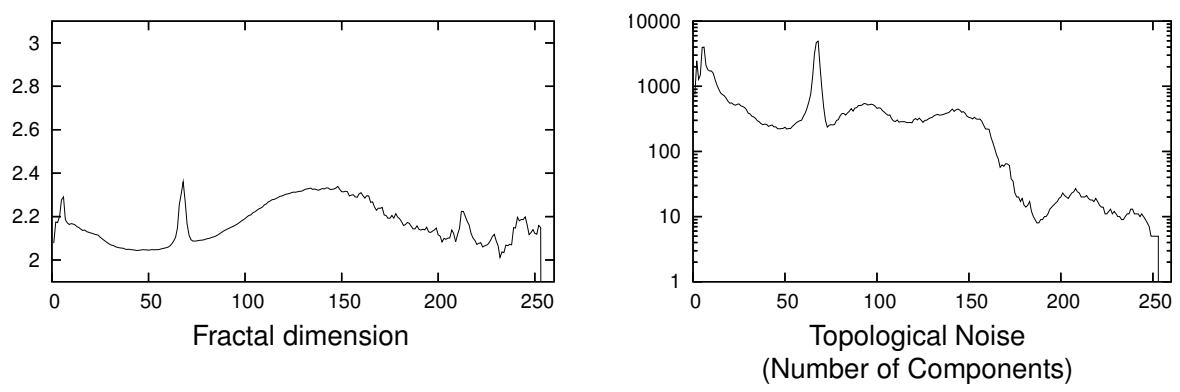

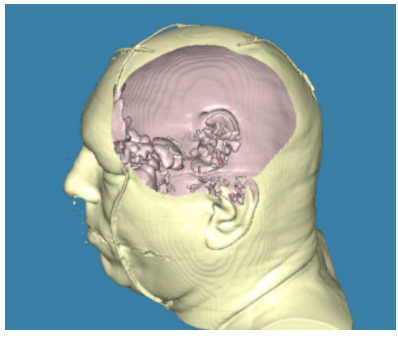

Isovalue 60

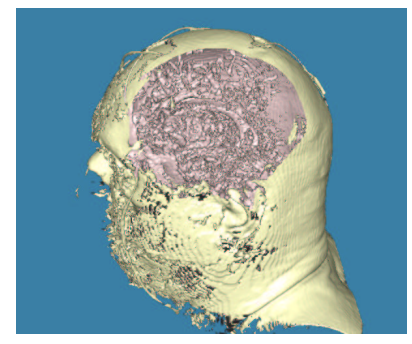

Isovalue 68

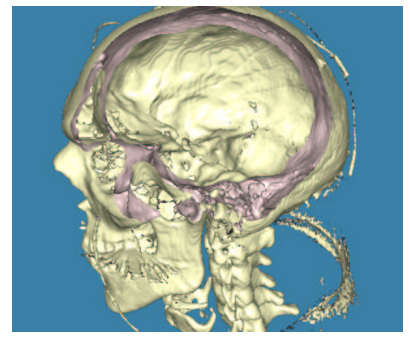

Isovalue 72

Fig. 1: Visible male data set (www.stereofx.org): Fractal box span dimension, number of isosurface components and sample isosurfaces. Noisy isosurface at isovalue 68 corresponds to high fractal dimension and large topological noise. Topological noise is measured by the number of connected components in the isosurface. (Visible male data set provided by the National Library of Medicine, USA.)

\begin{abstract}
A (3D) scalar grid is a regular $n_{1} \times n_{2} \times n_{3}$ grid of vertices where each vertex $v$ is associated with some scalar value $s_{v}$. Applying trilinear interpolation, the scalar grid determines a scalar function $g$ where $g(v)=s_{v}$ for each grid vertex $v$. An isosurface with isovalue $\sigma$ is a triangular mesh which approximates the level set $g^{-1}(\sigma)$. The fractal dimension of an isosurface represents the growth in the isosurface as the number of grid cubes increases. We define and discuss the fractal isosurface dimension. Plotting the fractal dimension as a function of the isovalues in a data set provides information about the isosurfaces determined by the data set. We present statistics on the average fractal dimension of 60 publicly available benchmark data sets. We also show the fractal dimension is highly correlated with topological noise in the benchmark data sets, measuring the topological noise by the number of connected components in the isosurface. Lastly, we present a formula predicting the fractal dimension as a function of noise and validate the formula with experimental results.
\end{abstract}

Index Terms-Isosurfaces, scalar data, fractal dimension.

\section{INTRODUCTION}

A (3D) scalar grid is a regular $n_{1} \times n_{2} \times n_{3}$ grid of vertices where each vertex $v$ is associated with some scalar value $s_{v}$. The grid covers some rectilinear region $\Omega$ and partitions $\Omega$ into cubes (or, more generally, rectilinear boxes.) Applying trilinear interpolation to each cube, gives a scalar function defined on each cube. Combining these scalar functions gives a scalar function $g: \Omega \rightarrow \mathbb{R}$ defined on $\Omega$. An isosurface

- Marc Khoury is an undergraduate and Dr. Rephael Wenger is an associate professor in the Computer and Information Science Department at The Ohio State University,

E-mails: khoury@cse.ohio-state.edu,wenger@cse.ohio-state.edu.

Manuscript received 31 March 2010; accepted 1 August 2010; posted online 24 October 2010; mailed on 16 October 2010.

For information on obtaining reprints of this article, please send e-mailto:tvcg@computer.org. with isovalue $\sigma$ is a triangular mesh which approximates the level set $g^{-1}(\sigma)$

Scalar grids can be created by scanning devices such as MRI or CT scanners, can be generated by numerical solutions of partial differential equations such as fluid flow simulations, or can be sampled from a scalar function $f: \mathbb{R}^{3} \rightarrow \mathbb{R}$. Increases in the resolution of the scanning device, the numerical simulation or the function sampling, permit increases in the size of the scalar grid representing the data. As the scalar grid grows, the size of the isosurface with isovalue $\sigma$ also grows. What is the relationship between the growth of the scalar grid and the growth of the isosurface? Specifically, if $N$ is the number of cubes in a scalar grid, how fast does the isosurface size grow as a function of $N$ ?

The size of an isosurface can be measured in terms of its area or the number of its triangles, but those measurements depend upon the specific approximation to $g^{-1}(\sigma)$. A better measurement is to count the number of grid cubes intersected by the isosurface. More specifically, count the number of grid cubes with span $[\alpha, \beta]$ such that $\alpha<\sigma \leq \beta$. 
For isosurfaces constructed by the Marching Cubes Algorithm and numerous variants, the number of grid cubes where $\alpha<\sigma \leq \beta$ is exactly the number of grid cubes intersected by the isosurface. For such isosurfaces, isosurface area and the number of isosurface triangles are directly proportional to the number of intersected grid cubes.

Let $X$ be a subset of a rectangular region $\Omega$. Let $I_{N}(X)$ be the number of grid cubes intersected by $X$ when $\Omega$ is covered by a grid of $N$ cubes with dimensions $\delta \times \delta \times \delta$. The fractal box counting dimension of $X$ is [7, Chapter 3]:

$$
\lim _{\delta \rightarrow 0} \frac{\log \left(I_{N}(X)\right)}{\log (1 / \delta)}
$$

The volume of $\Omega$ equals $N \delta^{3}$. Since this volume is constant, $\delta$ is proportional to $1 / N^{1 / 3}$. The fractal box counting dimension can be redefined as:

$$
\lim _{\delta \rightarrow 0} \frac{\log \left(I_{N}(X)\right)}{\log (1 / \delta)}=\lim _{N \rightarrow \infty} \frac{\log \left(I_{N}(X)\right)}{\log \left(N^{1 / 3}\right)}=\lim _{N \rightarrow \infty} 3 \frac{\log \left(I_{N}(X)\right)}{\log (N)} .
$$

The fractal box counting dimension represents the growth in the number of grid cubes intersected by $X$ as a function of the grid size. The fractal box counting dimension is one of a number of different ways to define fractal dimension, not all of them equivalent [7].

While isosurfaces are not simply subsets $X$ of $\Omega$, they are similar enough so that an equivalent fractal box counting dimension can be defined for isosurfaces. Instead of taking a limit as $N$ goes to infinity, we subsample the scalar grid and compare the number of intersected grid cubes of the full resolution and subsampled grid. In Section 3, we present a definition of the fractal box counting dimension of an isosurface and compare it with a previous definition of isosurface fractality in [17].

The fractal dimension is directly related to the growth in isosurface size. If the number of cubes intersected by an isosurface grows as $\Theta\left(N^{2 / 3}\right)$, then the fractal box counting dimension of the isosurface is two. If the number of intersected cubes grows as $\Theta(N)$, then the fractal dimension of the isosurface is three. More generally, if the number of intersected cubes grows as $\Theta\left(N^{\kappa}\right)$ where $2 / 3 \leq \kappa \leq 1$, then the fractal dimension is $3 \kappa$.

Using the techniques from [1] and [7], we can plot the fractal box counting dimension as a function of the isovalues in the data set. (See Figures 1 and 2.) Fractal dimensions near two suggest relatively smooth isosurfaces while higher fractal dimensions suggest rough isosurfaces with numerous small features.

If a scalar grid is a sampling of an algebraic function $f: \mathbb{R}^{3} \rightarrow \mathbb{R}$, then, for all isovalues, the fractal dimension is two. Similarly, if an isosurface is nearly planar, then its fractal dimension is also two [9].

However, what is the average fractal dimension of isosurfaces produced from benchmark data? A similar question was first asked in [4] and [18] which compared the isosurface sizes of different benchmark data sets. The conclusion in that paper was that the number of intersected cubes grows at a rate of $\Theta\left(N^{0.82}\right)$ for a fractal dimension of 2.46. A subsequent paper, [18], suggested an even higher growth rate of $\Theta\left(N^{0.96}\right)$ with a fractal dimension of 2.88. In this paper, we conclude that the average growth rate and average fractal dimension is substantially smaller.

As noted in [4] and [18], the isosurface fractal dimension increases with the addition of noise. In [18], synthetic noise is added to data sets and the increase in fractal dimension is measured and graphed. Is there a way to measure the noise in the benchmark data sets and correlate it to the fractal dimension?

One problem in isosurface construction is topological noise, small topological features such as small connected components, loops or tunnels, created by noise in the scalar data. Counting the number of connected components in an isosurface gives us some measure of this topological noise. We measured the number of such connected components and showed that it is correlated to the fractal dimension of the isosurface.

As shown from the synthetic experiments and the measurements of benchmark data sets, the fractal dimension increases with noise. What

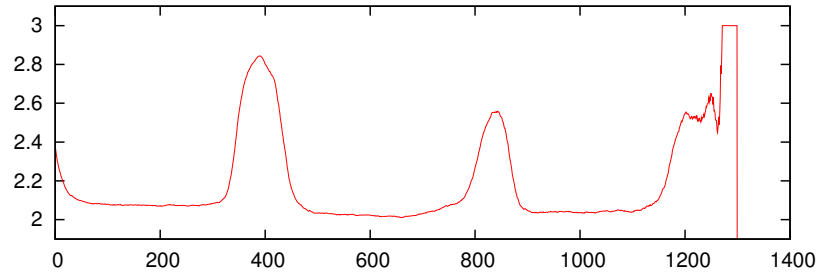

Fig. 2: Tooth data set (www.stereofx.org): Fractal box span dimension as a function of isovalue. (Tooth data set provided by GE Aircraft Engines, USA.)

exactly is the relationship between the two? In Section 5, we derive and present an analytic formula for the fractal dimension as a function of uniform noise added to a data set. We confirm the formula with experimental results.

The main contributions of this paper are as follows:

1. Definition of the fractal box counting dimension which can be easily computed and plotted as a function of scalar values;

2. Measurements of the isosurface growth and fractal dimension of benchmark data sets using grid subsampling;

3. Correlation of the fractal dimension with topological noise;

4. An analytic formula for the fractal dimension as a function of uniform noise.

\section{Previous Work}

The most popular algorithm for constructing isosurfaces from scalar data sets is the 1987 Marching Cubes Algorithm by Lorensen and Cline [11]. There are numerous variants $[2,3,12,14,16,19,20]$ and readers are referred to the survey paper [13] for a more complete listing. Other approaches to isosurface construction use dual contouring $[8,10,15]$ and Delaunay triangulations [6].

In a 1995 paper presenting a new technique for isosurface construction, Itoh and Koyamada noted that a nearly planar isosurface will intersect $\Theta\left(N^{2 / 3}\right)$ grid cubes [9]. They did not present any formal analysis or experimental evidence to justify this claim.

In 1997, Bajaj et. al. [1] proposed analyzing scalar data by measuring and plotting various isosurface statistics as a function of scalar values. They measured isosurface areas, volumes enclosed by isosurfaces and some gradient measures over the isosurface. Pekar et. al. in [17] showed how to quickly compute some of these gradient measures without explicitly computing the isosurface.

Let $G_{0}$ be a scalar grid with $N$ vertices and let $G_{1}, G_{2}, \ldots$ be subsampled versions of $G_{0}$ where $G_{k}$ has $(1 / 8)^{k} N$ vertices. Let $A^{\sigma}\left(G_{k}\right)$ be the area of an isosurface with isovalue $\sigma$ in $G_{k}$. Pekar et. al. [17] defined the fractal dimension of an isosurface as the slope of the linear regression of $\log \left(A^{\sigma}\left(G_{k}\right)\right)$. They did not make any conjectures or give any experimental data on the expected or average fractal dimension of isosurfaces. The definition of fractal isosurface dimension provided in this paper is different although similar to the one in [17].

In 2006, Carr et. al. [4] presented the first measurements on benchmark data sets of isosurface growth as a function of grid size $N$. They plotted isosurface size versus grid size for a set of ninety benchmark data sets and used least squares to fit a line to the data. Using the slope of the line, they estimated the growth rate as $\Theta\left(N^{0.82}\right)$. A subsequent paper by Scheidegger et. al.[18] corrected some errors in [4] and estimated a growth rate of $\Theta\left(n^{0.96}\right)$. They also experimented with adding synthetic noise to their data sets and measuring the growth rate as a function of the noise.

\section{Fractal Dimension}

As previously noted, there are numerous algorithms for isosurface construction and these algorithms produce numerous different, albeit 
Table 1: Measurements of fractal box dimension and correlation between fractal dimension and number of isosurface components. a) Type of data sets. Data sets of type "medical" are MRI and CT scans of humans. Data sets of type "measured" include non-organic data sets such as engine and organic data sets such as bonsai, monkey-CT and lobster. All other data sets are of type "synthetic", and include numerical simulation data such as bluntfin, neghip or shockwave and computer generated models such as hydrogenAtom, nucleon or silicium. b) Number of data sets. c) Average fractal dimension. (Average of the average fractal dimension of each data set.) d) Standard deviation of fractal dimension. e) Average of the standard deviation of the fractal dimension. (Average of the standard deviation of the fractal dimension of each data set.) f) Average fractal dimension of subsampled data sets. g) Average of the magnitude of the difference between the fractal dimension of the full resolution and subsampled version of each data set. $h$ ) Average correlation between the fractal dimension and the number of connected components for each data set. i) Standard deviation of the correlation.

\begin{tabular}{|c|c|c|c|c|c|c|c|c|}
\hline \multicolumn{2}{|c|}{ Data Sets } & \multicolumn{5}{|c|}{ Fractal Box Dim } & \multicolumn{2}{|c|}{ Correlation } \\
\hline a) Type & b) Num & c) Avg & d) Std Dev & e) Avg Std Dev & f) Avg Subsampled & g) Avg $\mid$ Full - Sub & h) Avg & i) Std Dev \\
\hline All & 60 & 2.26 & 0.16 & 0.13 & 2.38 & 0.14 & 0.61 & 0.18 \\
\hline Medical & 24 & 2.29 & 0.12 & 0.14 & 2.42 & 0.13 & 0.65 & 0.11 \\
\hline $\begin{array}{l}\text { Measured } \\
\text { (non-medical) }\end{array}$ & 22 & 2.26 & 0.17 & 0.13 & 2.39 & 0.15 & 0.61 & 0.19 \\
\hline Synthetic & 14 & 2.20 & 0.22 & 0.09 & 2.30 & 0.13 & 0.52 & 0.25 \\
\hline
\end{tabular}

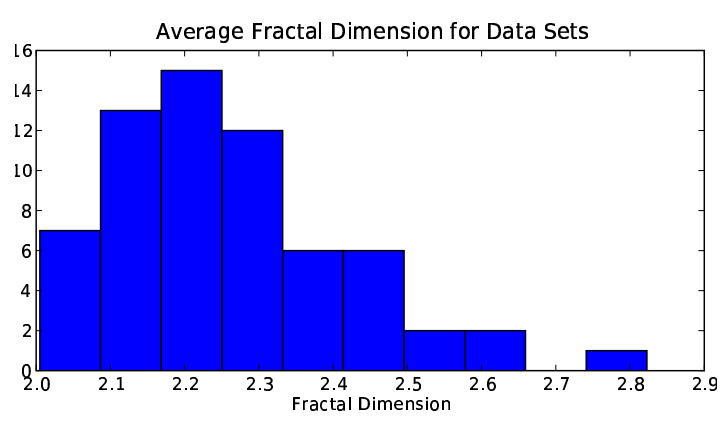

Fig. 3: Histogram of average fractal dimensions for 60 benchmark data sets.

similar, isosurfaces. Since the sizes of these isosurfaces vary by only a constant factor, they all have the same asymptotic growth rate.

The span of a grid cube $C$ is a closed interval $[\alpha, \beta]$ where $\alpha$ is the smallest scalar value of any cube vertex and $\beta$ is the largest. If an isovalue $\sigma$ is strictly between $\alpha$ and $\beta$, then any isosurface with isovalue $\sigma$ will intersect $C$. (If $\sigma$ equals $\alpha$ or $\beta$, then the isosurface may or may not intersect $C$.) For a scalar grid $G$, let $I^{\sigma}(G)$ be the number of cubes whose span contains $\sigma$.

Let $f: \Omega \rightarrow \mathbb{R}$ be a scalar function defined on a rectangular region $\Omega$. Function $f$ is not necessarily continuous. Let $G_{N}$ be a scalar grid with $N$ cubes covering $\Omega$ which samples $f$ at its vertices. Define the fractal box span dimension of $f$ for isovalue $\sigma$ as:

$$
\lim _{N \rightarrow \infty} \frac{\log \left(I^{\sigma}\left(G_{N}\right)\right)}{\log \left(N^{1 / 3}\right)}=\lim _{N \rightarrow \infty} 3 \frac{\log \left(I^{\sigma}\left(G_{N}\right)\right)}{\log (N)} .
$$

(If this limit does not exist, then we should use upper and lower limits.)

The fractal box span dimension is similar to the fractal box counting dimension defined in the introduction. However, it is not necessarily the same as the fractal box counting dimension of the level set $f^{-1}(\sigma)$. If $f$ is not continuous, there may be no point $p$ for which $f(p)$ equals $\sigma$ and yet an infinite number of arbitrarily close pairs of points $(p, q)$ such that $f(p) \leq \sigma \leq f(q)$.

A standard technique to measure the fractal box counting dimension of a point set $X$ is to plot $3 \log \left(I_{N}(X)\right)$ as a function of $\log (N)$, use linear regression to fit a line to the plot, and then take the slope of that line. Similarly, Pekar et. al. [17] measure the fractal box span dimension by plotting $3 \log \left(A^{\sigma}\left(G_{N}\right)\right)$ as a function of $\log (N)$, and taking the slope of the line fitting that plot. (Pekar et. al. used $A^{\sigma}\left(G_{N}\right)$, the area of the isosurface with isovalue $\sigma \in G_{N}$, instead of $I^{\sigma}\left(G_{N}\right)$.)

If we take just two regular grids, $G_{N}$ and $G_{N / 8}$, where $G_{N / 8}$ has $N / 8$ cubes, then the slope of the line through $\left(\log (N / 8), 3 \log \left(I^{\sigma}\left(G_{N / 8}\right)\right)\right)$ and $\left(\log (N), 3 \log \left(I^{\sigma}\left(G_{N}\right)\right)\right)$ is:

$$
\frac{3 \log \left(I^{\sigma}\left(G_{N}\right)\right)-3 \log \left(I^{\sigma}\left(G_{N / 8}\right)\right)}{\log (N)-\log (N / 8)}=\frac{\log \left(I^{\sigma}\left(G_{N}\right)\right)-\log \left(I^{\sigma}\left(G_{N / 8}\right)\right)}{\log (2)} .
$$

Using base two for the log, simplifies this to:

$$
\log _{2}\left(I^{\sigma}\left(G_{N}\right)\right)-\log _{2}\left(I^{\sigma}\left(G_{N / 8}\right)\right)=\log _{2}\left(\frac{I^{\sigma}\left(G_{N}\right)}{I^{\sigma}\left(G_{N / 8}\right)}\right) .
$$

Let $G$ be a scalar grid with $N$ cubes and let $G^{\prime}$ be the subsampled regular grid of $G$ with $N / 8$ cubes. We define the fractal box span dimension of scalar grid $G$ for isovalue $\sigma$ as:

$$
\log _{2}\left(\frac{I^{\sigma}(G)}{I^{\sigma}\left(G^{\prime}\right)}\right)
$$

Subsampling a data set usually involves using nearby data points such as in a Gaussian filter to construct as good an estimate as possible of the remaining scalar values. However, doing so implicitly smooths the data. It raises the possibility that the differences between $G$ and $G^{\prime}$ measured by the fractal dimension are caused by the better estimation of scalar values in $G^{\prime}$, not an inherent growth in the isosurface area. Using a filter also begs the question of which filter and which filter parameters to use. Subsampling in this paper always refers to the simple selection of every other point in the data set without any application of a filter.

We measured the fractal box span dimension of 60 public benchmark data sets from the two web sites www. stereofx.org and www.volvis.org. Each data set represented a scalar grid. For all the data sets, scalar values at grid vertices were 8 bit or 12 bit integers. For each data set, we computed the fractal box span dimension for each isovalue between the minimum and maximum scalar value in the data set. (See Figure 2.) We averaged the fractal dimension over the isovalues to compute an average fractal dimension for the data sets and then computed an average over all the data sets. (See Figure 3 and Table 1.) The average fractal dimension for all the data sets is 2.26 (standard deviation 0.16). We also computed the standard deviation of the fractal dimension of each data set and the average of those standard deviations. (See column e) in Table 1.) The average standard deviation is 0.13 .

Average fractal dimension 2.26 indicates that the isosurface grows as $\Theta\left(N^{0.75}\right)$ which is significantly less than the previously estimated $\Theta\left(N^{0.82}\right)$ [4] or $\Theta\left(N^{0.96}\right)$ [18]. As suggested in [4] and [18], we partitioned the data sets into three categories, medical, measured but nonmedical and synthetic. In [18], the isosurface growth was reported as $\Theta\left(N^{0.70}\right), \Theta\left(N^{0.82}\right)$ and $\Theta\left(N^{0.87}\right)$ for medical, measured (nonmedical) and synthetic data sets. Our corresponding measurements are $\Theta\left(N^{0.76}\right), \Theta\left(N^{0.75}\right)$ and $\Theta\left(N^{0.73}\right)$. 


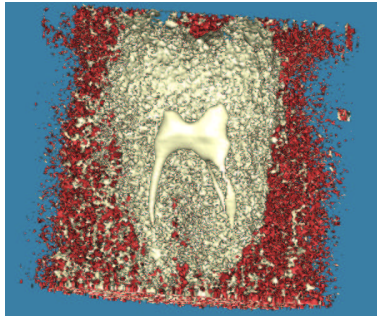

Isovalue 400

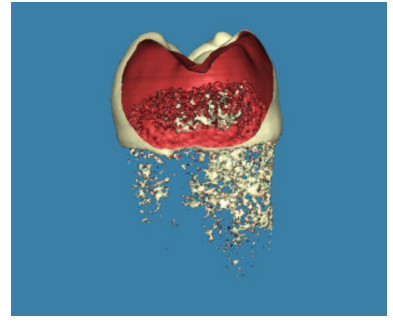

Isovalue 850
Fig. 4: Noisy isosurfaces from the Tooth data set.

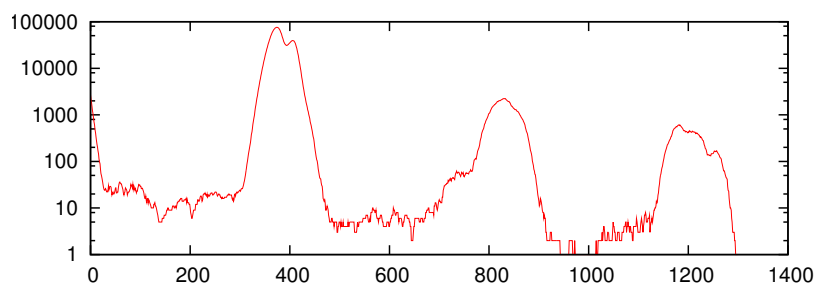

Fig. 5: Tooth data set: Number of isosurface components as a function of isovalue (log scale).

Our definition of fractal dimension uses only two grids to estimate the fractal dimension as opposed to the multiple subgrids suggested by Pekar et. al. Our definition has the advantage that it is a precise formula. We do not have to specify the number of subgrids and/or the criterion and algorithm used to fit a line to the plot. Our definition also uses the number of grid cubes whose span contains the isovalue instead of isosurface area. This definition is simple and precise, does not depend upon an isosurface construction algorithm, and does not require constructing an isosurface.

The fractal box span dimension of a scalar grid is meant to approximate an infinite sequence of scalar grids. To see how well it does so, we subsampled the scalar grid in the 60 benchmark data sets using one-eighth the number of vertices, and then measured the average fractal dimension of the subsampled grids. The average fractal box dimension of the subsampled grids is 2.38 (standard deviation 0.16). The average magnitude of the difference between the subsampled and the full resolution fractal dimension is 0.15 (standard deviation 0.08.) Note that the fractal box span dimension of a subsampled grid $G^{\prime}$ depends upon $I^{\sigma}\left(G^{\prime \prime}\right)$ where $G^{\prime \prime}$ is a subsampled grid of $G^{\prime}$. Grid $G^{\prime \prime}$ has $1 / 64$ the vertices of the original grid, which is a significant subsampling of the original grid.

\section{Correlating Fractal Dimension and Noise}

As demonstrated in Figure 2, the fractal box span dimension often varies greatly with the isovalue. On closer inspection, isosurfaces with large fractal dimensions are greatly affected by noise in the scalar data. For instance, the isovalues 400 and 850 in Figure 2 with high fractal dimensions correspond to the noisy isosurfaces in Figure 4.

Noise in a scalar data set is a set of random values under some probability distribution which is added to the scalar values of the grid vertices. The noise may have various frequencies, although it is the high frequency noise which affects the fractal box span dimension.

In [18], Scheidegger et. al. added computer generated noise to scalar data generated from an algebraic equation and showed that the fractal box span dimension increased as the noise amplitude increased. While this experiment shows that noise can cause a high fractal box span dimension, it does not necessarily follow that the high fractal dimension measured in the benchmark data sets is caused by noise.

To show that noise is at least partially the cause of the high fractal dimension in benchmark data sets, we need some measure of noise. Topological noise in an isosurface is topological isosurface features, such as connected components, loops or tunnels, created by noise in

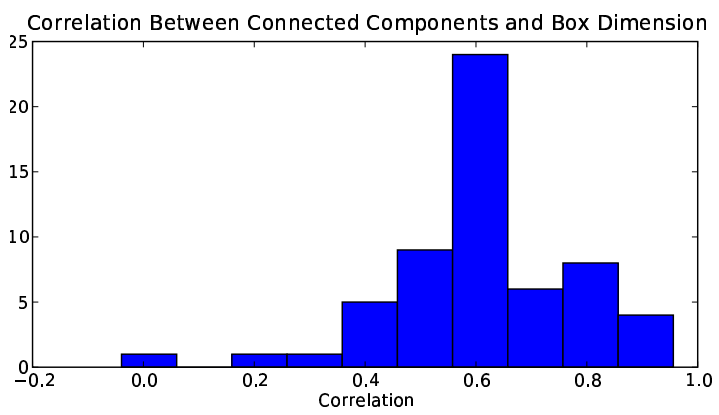

Fig. 6: Histogram of correlation between average fractal dimension and number of isosurface components for 65 benchmark data sets.

the scalar data. We measured the noise in the data set by measuring the number of connected components in the isosurface. Assuming the data sets represent surfaces with a dozen or fewer components, most of the components represent noise.

For a given data set, the number of isosurface components and the fractal box span dimension vary with the isovalue. How well are those two values correlated? For instance, in Figure 5 the large number of components for isovalues 400 and 850 correspond to the high fractal box span dimension for those values shown in Figure 2.

The number of isosurface components is dependent upon noise but it is also dependent upon the isosurface area. To correct for this dependency upon isosurface area, we divided the number of isosurface components by the area and correlated the resulting number with the fractal dimension. We approximated the isoarea by the number of edges intersected by the isosurface. We ignored isovalues for isosurfaces which had very small area since such small isosurfaces biased the result. More specifically, for each data set we took the size of the smallest two dimensional facet of the grid and divided by four. Isosurfaces which intersected fewer than that number of grid edges were considered insignificantly small, and their isovalues were ignored. Correlation statistics are presented in Table 1 and Figure 6.

To measure the number of connected isosurface components, we used the algorithm by Carr et. al. [5] for constructing contour trees. Each contour tree edge represents a set of connected isosurface components which vary with the isovalue. For each isovalue, we counted the number of contour tree edges which represent an isosurface component with that isovalue. This number equals the number of connected components of an isosurface with that isovalue.

Some data sets had average fractal dimension very close to two with very little variance as a function of isovalue. We ignored the four data sets whose standard deviation of the fractal dimension was less than 0.05 . The average correlation coefficient for the remaining data sets was 0.61 (standard deviation 0.18 ).

\section{Fractal Dimension as a Function of Noise}

As Scheidegger et. al. [18] showed, the fractal box span dimension increases with noise. What exactly is the relationship between the two?

Computing the probability that a grid cube is intersected by the isosurface is complicated, since it depends on the scalar values of eight grid vertices. Instead of using grid cubes, we use grid edges. The span of a grid edge $e$ is a closed interval $[\alpha, \beta]$ where $\alpha$ and $\beta$ are the scalar values of the grid endpoints and $\alpha \leq \beta$. For a scalar grid $G$, let $I_{e}^{\sigma}(G)$ be the number of edges whose span contains $\sigma$.

Let $G$ be a scalar grid with $N$ vertices and let $G^{\prime}$ be the subsampled regular grid of $G$ with $N / 8$ vertices. Define the fractal edge span dimension of $G$ for isovalue $\sigma$ as:

$$
\log _{2}\left(\frac{I_{e}^{\sigma}(G)}{I_{e}^{\sigma}\left(G^{\prime}\right)}\right) .
$$

As shown in Figure 7, the fractal edge span and box span dimensions are similar but not equivalent. 
Let $G$ be a scalar grid. Each grid vertex $v_{a}$ of $G$ has a scalar value $s_{a}$. For each grid vertex $v_{a}$ of $G$, create a "noisy" scalar value, $\tilde{s}_{a}$, which is $s_{a}$ plus a random value with uniform distribution in the range $[-\mu, \mu]$. Let $\widetilde{G}$ be the scalar grid created by replacing the scalar value $s_{a}$ with $\tilde{s}_{a}$ for each vertex $v_{a}$ of $G$. We wish to give a formula for the fractal dimension of $\widetilde{G}$ as a function of $\mu$, the range of the noise.

To make any predictions about the fractal dimension of an isosurface, we need some conditions on the grid edges in the neighborhood of that isosurface. If edge $e$ has edge span $\left[\alpha_{e}, \beta_{e}\right]$, (where $\alpha_{e} \leq \beta_{e}$, by convention,) then the minimum scalar value of edge $e$ is $\alpha_{e}$. The magnitude of the span of $e$ is $\left|\beta_{e}-\alpha_{e}\right|$. Define a bivariate function $\Psi$ on the grid edges as:

$$
\Psi(e)=\left(\Psi_{1}(e), \Psi_{2}(e)\right)=\left(\alpha_{e},\left|\beta_{e}-\alpha_{e}\right|\right) .
$$

Let $\sigma$ be an isovalue and $E_{\sigma}(\eta)$ be the edges whose minimum scalar values are in the range $[\sigma-\eta, \sigma+\eta]$. Our formula will be based on the assumption that $\Psi$ restricted to $E_{\sigma}(\eta)$ approximates a bivariate uniform distribution where $\Psi_{1}(e)$ varies uniformly in the range $[\sigma-\eta, \sigma+\eta]$ and $\Psi_{2}(e)$ varies uniformly from 0 to $\gamma$.

The simplest example of a scalar grid with this property is a regular grid of unit cubes whose scalar values are sampled from a point cloud. Let $f: \mathbb{R}^{3} \rightarrow \mathbb{R}$ represent the Euclidean distance to the origin and let $G$ be a regular grid of cubes centered at the origin. The gradient magnitude of $f$ is one. The vertices of $G$ uniformly sample the region between the concentric spheres $f^{-1}(\sigma-\eta)$ and $f^{-1}(\sigma+\eta)$. Within this region, the gradient directions vary uniformly over the unit sphere. Projecting these gradients onto the $\mathrm{x}$-axis, the projections vary uniformly from -1 to 1 . (The area of a sphere cap with height $h$ is $2 \pi h$ so the probability that the projection is in the range $[-1,-1+h]$ is $2 \pi h /(4 \pi)=h / 2$.) These projections determine the magnitudes of the spans of edges parallel to the $x$-axis. Thus the magnitude of these spans vary uniformly from 0 to 1 . Similarly, the magnitude of the spans of edges parallel to the $y$ and $z$ axis vary uniformly from 0 to 1 .

While grid $G$ uniformly samples the region between $f^{-1}(\sigma-\eta)$ and $f^{-1}(\sigma+\eta)$, the volume between $f^{-1}(\sigma-\eta)$ and $f^{-1}(\sigma)$ is greater than the volume between $f^{-1}(\sigma)$ and $f^{-1}(\sigma+\eta)$. Thus, $\Psi_{1}$ restricted to $E_{\sigma}(\eta)$ does not vary uniformly in the range $[\sigma-\eta, \sigma+$ $\eta]$. When $\sigma$ is much larger than $\eta$, the difference between these volumes is small and the distribution of minimum scalar values in $E_{\sigma}(\eta)$ is nearly, although not quite, uniform. Thus, $\Psi$ restricted to $E_{\sigma}(\eta)$ approximates a bivariate uniform distribution only if $\sigma$ is much larger than $\eta$.

Instead of the distance to one point, grid $G$ could sample the distance function to a finite set of random points. Gradient magnitudes of this function are constant and with high probability the gradient directions vary nearly uniformly. When $\sigma$ is much larger than $\eta$, function $\Psi$ restricted to $E_{\sigma}(\eta)$ approximates a bivariate uniform distribution.

Let $E_{\sigma}^{\prime}(\eta)$ be the edges of the subsampled grid $G^{\prime}$ whose minimum scalar values are in the range $[\sigma-\eta, \sigma+\eta]$. The following theorem relates the fractal dimension to noise under the appropriate conditions:

Theorem 1. Let $r$ and $\gamma$ be non-negative real numbers and let $\eta$ equal $(r+2) \gamma$. Let $G$ be a regular grid and $\sigma$ be an isovalue such that $\Psi$ restricted to $E_{\sigma}(\eta)$ and $\Psi$ restricted to $E_{\sigma}^{\prime}(\eta)$ approximates the bivariate uniform distribution on $[\sigma-\eta, \sigma+\eta] \times[0, \gamma]$ and where $\left|E_{\sigma}^{\prime}(\eta)\right|$ approximately equals $\left|E_{\sigma}(\eta)\right| / 8$. Let $\widetilde{G}$ be a scalar grid created by adding random noise with uniform distribution in the range $[-r \gamma, r \gamma]$ to $G$.

1. If $r \geq 1$, then the expected fractal edge span dimension of $\widetilde{G}$ is:

$$
\log _{2}\left(\frac{32 r^{3}+8 r-1}{32(r / 2)^{3}+8(r / 2)-1}\right) .
$$

2. If $1 / 2 \leq r \leq 1$, then the expected fractal edge span dimension of $\widetilde{G}$ is:

$$
\log _{2}\left(\frac{32 r^{3}+8 r-1}{r^{4}+6 r^{2}}\right)
$$

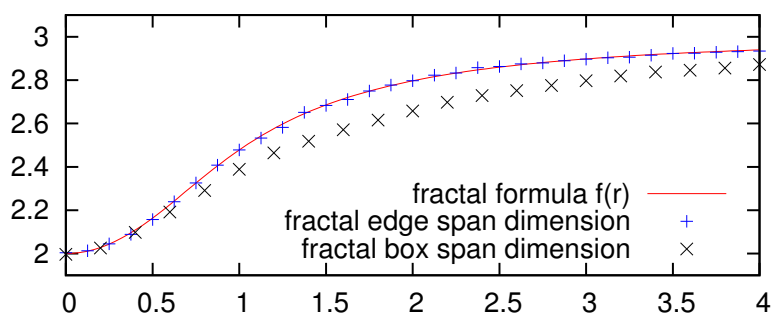

Fig. 7: Plot of fractal formula (Equations 1, 2 and 3.) Function $f(r)$ represents the fractal edge span dimension as a function of uniform noise in the range $[-r \gamma, r \gamma]$ where $\gamma$ is the gradient. Plot of fractal edge span dimension and fractal box span dimension versus noise added to a point cloud data set.

3. If $r \leq 1 / 2$, then the expected fractal edge span dimension of $\widetilde{G}$ is:

$$
\log _{2}\left(4 \frac{2 r^{2}+3}{2(r / 2)^{2}+3}\right)
$$

Setting $r$ to zero means no noise is added to the data set. In that case, Equation 3 gives the expected fractal dimension of $G$ as two. Thus the formula represents the growth in fractal dimension as a function of noise. The fractal formula (Equations 1,2 and 3) is graphed in Figure 7.

To derive the formula, we first compute the probability that an edge $\left(v_{a}, v_{b}\right)$ in $\widetilde{G}$ intersects the isosurface (Lemma 1). This probability depends upon the scalar values of $v_{a}$ and $v_{b}$ in the original scalar grid $G$. We next compute the probability that an edge whose gradient span is $\delta$ intersects the isosurface (Lemma 2). We do so by integrating over all minimum scalar values of edges with span magnitude $\delta$. Finally, we integrate over the span magnitude $\delta$ to compute the probability that a random edge intersects the isosurface (Lemma 3). We compute the expected number of edges in $\widetilde{G}$ and its subsampling $\widetilde{G}^{\prime}$ which intersect the isosurface and compute the fractal dimension from the expected number of intersections.

Let $\left(v_{a}, v_{b}\right)$ be a grid edge in $\widetilde{G}$. An isosurface with isovalue $\sigma$ in $\widetilde{G}$ intersects edge $\left(v_{a}, v_{b}\right)$ if $\sigma \in\left[\tilde{s}_{a}, \tilde{s}_{b}\right]$. (Note that we make no assumptions about whether $\tilde{s}_{a}$ or $\tilde{s}_{b}$ is greater.)

Lemma 1. Let $\widetilde{G}$ be a scalar grid created by adding random noise with uniform distribution in the range $[-\mu, \mu]$ to $G$. Let $\left(v_{a}, v_{b}\right)$ be a grid edge of $G$ where $v_{a}$ has scalar value $s_{a}$ in $G$ and $\tilde{s}_{a}$ in $\widetilde{G}$ and $v_{b}$ has scalar value $s_{b}$ in $G$ and $\tilde{s}_{b}$ in $\widetilde{G}$.

1. If $\sigma \in \mathbb{R}$ is a scalar value where $\sigma-\mu \leq s_{a} \leq s_{b} \leq \sigma+\mu$, then the probability that $\sigma \in\left[\tilde{s}_{a}, \tilde{s}_{b}\right]$ is: $\frac{1}{2}-\frac{\left(s_{a}-\sigma\right)\left(s_{b}-\sigma\right)}{2 \mu^{2}}$.

2. If $\sigma \in \mathbb{R}$ is a scalar value where $s_{a} \leq \sigma-\mu \leq s_{b} \leq \sigma+\mu$, then the probability that $\sigma \in\left[\tilde{s}_{a}, \tilde{s}_{b}\right]$ is: $\frac{1}{2}+\frac{s_{b}-\sigma}{2 \mu}$.

3. If $\sigma \in \mathbb{R}$ is a scalar value where $\sigma-\mu \leq s_{a} \leq \sigma+\mu \leq s_{b}$, then the probability that $\sigma \in\left[\tilde{s}_{a}, \tilde{s}_{b}\right]$ is: $\frac{1}{2}+\frac{s_{a}-\sigma}{2 \mu}$.

Proof. If $\sigma-\mu \leq s_{a} \leq \sigma+\mu$, then the probability that $\tilde{s}_{a}$ is greater than $\sigma$ is:

$$
P\left(\tilde{s}_{a} \geq \sigma\right)=\frac{\mu+s_{a}-\sigma}{2 \mu}=\frac{1}{2}+\frac{s_{a}-\sigma}{2 \mu} .
$$

Since $s_{a}$ is in the range $[\sigma-\mu, \sigma+\mu]$, the value on the right is always between 0 and 1 . The probability that $s_{a}$ is less than $\sigma$ is:

$$
P\left(\tilde{s}_{a} \leq \sigma\right)=\frac{\mu-\left(s_{a}-\sigma\right)}{2 \mu}=\frac{1}{2}-\frac{s_{a}-\sigma}{2 \mu} .
$$


Similar probabilities hold for $s_{b}$ and $\tilde{s}_{b}$.

The probability that $\sigma \in\left[\tilde{s}_{a}, \tilde{s}_{b}\right]$ is the probability that $\tilde{s}_{a} \leq \sigma \leq \tilde{s}_{b}$ or that $\tilde{s}_{a} \geq \sigma \geq \tilde{s}_{b}$. Since these two events are mutually exclusive, (except for the zero probability event that $\tilde{s}_{a}=\tilde{s}_{b}=\sigma$,) the probability that $\sigma \in\left[\tilde{s}_{a}, \tilde{s}_{b}\right]$ is the sum of these two probabilities.

Case 1: $\sigma-\mu \leq s_{a} \leq s_{b} \leq \sigma+\mu$.

$$
\begin{aligned}
P\left(\sigma \in\left[\tilde{s}_{a}, \tilde{s}_{b}\right]\right) & =P\left(\tilde{s}_{a} \leq \sigma \leq \tilde{s}_{b}\right)+P\left(\tilde{s}_{a} \geq \sigma \geq \tilde{s}_{b}\right) \\
& =\frac{1}{2}-\frac{\left(s_{a}-\sigma\right)\left(s_{b}-\sigma\right)}{2 \mu^{2}} .
\end{aligned}
$$

Case 2: $s_{a} \leq \sigma-\mu \leq s_{b} \leq \sigma+\mu$.

The probability that $\tilde{s}_{a} \geq \sigma$ is zero. Thus,

$$
P\left(\sigma \in\left[\tilde{s}_{a}, \tilde{s}_{b}\right]\right)=P\left(\tilde{s}_{a} \leq \sigma \leq \tilde{s}_{b}\right)=\frac{1}{2}+\frac{s_{b}-\sigma}{2 \mu} .
$$

Case 3: $\sigma-\mu \leq s_{a} \leq \sigma+\mu \leq s_{b}$.

The probability that $\tilde{s}_{b} \leq \sigma$ is zero. Thus,

$$
P\left(\sigma \in\left[\tilde{s}_{a}, \tilde{s}_{b}\right]\right)=P\left(\tilde{s}_{a} \leq \sigma \leq \tilde{s}_{b}\right)=\frac{1}{2}+\frac{s_{a}-\sigma}{2 \mu} .
$$

To compute the probability that a random edge with span $[x, x+\delta]$ intersects the isosurface, we integrate over all the possible values of $x$. Let $\operatorname{span}_{\widetilde{G}}(e)$ be the span of edge $e$ in $\widetilde{G}$. Let $E(G)$ be the set of edges of $G$.

Lemma 2. Let $\widetilde{G}$ be a scalar grid created by adding random noise with uniform distribution in the range $[-\mu, \mu]$ to $G$. Let e be a random grid edge with span $[x, x+\delta]$ chosen from some $E^{\prime} \subseteq E(G)$ such that $x$ is uniformly distributed over $[\sigma-\Lambda, \sigma+\Lambda]$ for some $\Lambda \geq \mu+\delta$.

1. If $\delta \leq 2 \mu$, then the probability that $\sigma \in \operatorname{span}_{\widetilde{G}}(e)$ is

$$
\frac{8 \mu^{3}-\delta^{3}}{24 \mu^{2} \Lambda}+\frac{\delta^{2}}{4 \mu \Lambda}
$$

2. If $\delta \geq 2 \mu$, then the probability that $\sigma \in \operatorname{span}_{\widetilde{G}}(e)$ is $\delta /(2 \Lambda)$.

Proof of Statement 1: Applying Lemma 1, Statement 1, the probability that $\sigma \in \operatorname{span}_{\widetilde{G}}(e)$ given that $\sigma-\mu \leq x \leq x+\delta \leq \sigma+\mu$ is:

$$
\begin{aligned}
& \frac{1}{2 \mu-\delta} \int_{x=\sigma-\mu}^{\sigma+\mu-\delta}\left(\frac{1}{2}-\frac{(x-\sigma)(x+\delta-\sigma)}{2 \mu^{2}}\right) d x \\
& \quad=\frac{1}{2 \mu-\delta}\left(\frac{2}{3} \mu-\frac{\delta^{3}}{12 \mu^{2}}\right)=\frac{1}{2 \mu-\delta}\left(\frac{8 \mu^{3}-\delta^{3}}{12 \mu^{2}}\right) .
\end{aligned}
$$

The probability that $\sigma-\mu \leq x \leq x+\delta \leq \sigma+\mu$ is $(2 \mu-\delta) /(2 \Lambda)$. The probability that $\sigma-\mu \leq x \leq x+\delta \leq \sigma+\mu$ and $\sigma \in \operatorname{span}_{\widetilde{G}}(e)$ is:

$$
\left(\frac{2 \mu-\delta}{2 \Lambda}\right)\left(\frac{1}{2 \mu-\delta}\right)\left(\frac{8 \mu^{3}-\delta^{3}}{12 \mu^{2}}\right)=\frac{8 \mu^{3}-\delta^{3}}{24 \mu^{2} \Lambda} \text {. }
$$

Applying Lemma 1, Statement 2, the probability that $\sigma \in \operatorname{span}_{\widetilde{G}}(e)$ given that $x \leq \sigma-\mu \leq x+\delta \leq \sigma+\mu$ is:

$$
\frac{1}{\delta} \int_{x=\sigma-\mu-\delta}^{\sigma-\mu}\left(\frac{1}{2}+\frac{x+\delta-\sigma}{2 \mu}\right) d x=\frac{\delta}{4 \mu} .
$$

The probability that $x \leq \sigma-\mu \leq x+\delta \leq \sigma+\mu$ and $\sigma \in \operatorname{span}_{\widetilde{G}}(e)$

$$
\left(\frac{\delta}{2 \Lambda}\right)\left(\frac{\delta}{4 \mu}\right)=\frac{\delta^{2}}{8 \mu \Lambda}
$$

Similarly, the probability that $\sigma-\mu \leq x \leq \sigma+\mu \leq x+\delta$ and $\sigma \in$ $\operatorname{span}_{\widetilde{G}}(e)$ is also $\delta^{2} /(8 \mu \Lambda)$.

Adding the three cases, $\sigma-\mu \leq x \leq x+\delta \leq \sigma+\mu$ and $x \leq \sigma-\mu \leq$ $x+\delta \leq \sigma+\mu$ and $\sigma-\mu \leq x \leq \sigma+\mu \leq x+\delta$, the probability that $\sigma \in \operatorname{span}_{\widetilde{G}}(e)$ is:

$$
\frac{8 \mu^{3}-\delta^{3}}{24 \mu^{2} \Lambda}+\frac{\delta^{2}}{8 \mu \Lambda}+\frac{\delta^{2}}{8 \mu \Lambda}=\frac{8 \mu^{3}-\delta^{3}}{24 \mu^{2} \Lambda}+\frac{\delta^{2}}{4 \mu \Lambda} .
$$

Proof of Statement 2: If $\sigma$ is in $\operatorname{span}_{\widetilde{G}}(e)$, then $x$ must be in the range $[\sigma-\mu-\delta, \sigma+\mu]$. We divide this range into three cases:

$x \in[\sigma-\mu-\delta, \sigma+\mu-\delta]$ and $x \in[\sigma+\mu-\delta, \sigma-\mu]$ and $x \in[\sigma-\mu, \sigma+\mu]$.

Applying Lemma 1, Statement 2, the probability that $\sigma \in \operatorname{span}_{\widetilde{G}}(e)$ given that $\sigma-\mu-\delta \leq x \leq \sigma+\mu-\delta$ is:

$$
\frac{1}{2 \mu} \int_{x=\sigma-\mu-\delta}^{\sigma+\mu-\delta}\left(\frac{1}{2}+\frac{x+\delta-\sigma}{2 \mu}\right) d x=\frac{1}{2} .
$$

The probability that $\sigma-\mu-\delta \leq x \leq \sigma+\mu-\delta$ and $\sigma \in \operatorname{span}_{\widetilde{G}}(e)$ is $\mu /(2 \Lambda)$.

The probability that $\sigma \in \operatorname{span}_{\widetilde{G}}(e)$ given that $\sigma+\mu-\delta \leq x \leq \sigma-\mu$ is 1 since $\mu \leq \delta / 2$. The probability that $\sigma+\mu-\delta \leq x \leq \sigma-\mu$ and $\sigma \in \operatorname{span}_{\widetilde{G}}(e)$ is $(\delta-2 \mu) /(2 \Lambda)$.

The probability that $\sigma-\mu \leq x \leq \sigma+\mu$ and $\sigma \in \operatorname{span}_{\widetilde{G}}(e)$ is symmetric to the first case and is also $\mu /(2 \Lambda)$.

Summing the three cases, the total probability that $\sigma \in \operatorname{span}_{\widetilde{G}}(e)$ is $\delta /(2 \Lambda)$.

To compute the probability that a random edge $e$ intersects the isosurface, we integrate over the magnitude of edge spans.

Lemma 3. Let $\widetilde{G}$ be a scalar grid created by adding random noise with uniform distribution in the range $[-\mu, \mu]$ to $G$. Let e be a random grid edge with span $[x, x+\delta]$ chosen from $E^{\prime} \subseteq E(G)$ such that $\Psi$ restricted to $E^{\prime}$ has bivariate uniform distribution on $[\sigma-\Lambda, \sigma+\Lambda] \times$ $[0, \gamma]$ for some $\Lambda \geq \mu+\gamma$.

1. If $\gamma \leq 2 \mu$, then the probability that $\sigma \in \operatorname{span}_{\widetilde{G}}(e)$ is:

$$
\frac{32 \mu^{3}+8 \mu \gamma^{2}-\gamma^{3}}{96 \mu^{2} \Lambda}
$$

2. If $\gamma \geq 2 \mu$, then the probability that $\sigma \in \operatorname{span}_{\widetilde{G}}(e)$ is:

$$
\frac{3 \gamma^{2}+2 \mu^{2}}{12 \gamma \Lambda}
$$

Proof of Statement 1: Applying Lemma 2 and integrating over $\delta$, the probability that $\sigma \in \operatorname{span}_{\widetilde{G}}(e)$ is:

$$
\frac{1}{\gamma} \int_{\delta=0}^{\gamma}\left(\frac{8 \mu^{3}-\delta^{3}}{24 \mu^{2} \Lambda}+\frac{\delta^{2}}{4 \mu \Lambda}\right) d \delta=\frac{32 \mu^{3}+8 \mu \gamma^{2}-\gamma^{3}}{96 \mu^{2} \Lambda}
$$

Proof of Statement 2: We integrate the probability that $\sigma \in \operatorname{span}_{\widetilde{G}}(e)$ over $\delta$, splitting the integrand into two cases, one where $\delta \leq 2 \mu$ and the other where $\delta \geq 2 \mu$. Applying Lemma 2, the probability is:

$$
\begin{aligned}
& \frac{1}{\gamma}\left(\int_{\delta=0}^{2 \mu}\left(\frac{8 \mu^{3}-\delta^{3}}{24 \mu^{2} \Lambda}+\frac{\delta^{2}}{4 \mu \Lambda}\right) d \delta+\int_{\delta=2 \mu}^{\gamma}\left(\frac{\delta}{2 \Lambda}\right) d \delta\right) \\
& =\frac{1}{2 \gamma}\left(\frac{7 \mu^{2}}{3 \Lambda}+\frac{\gamma^{2}}{2 \mu}-\frac{2 \mu^{2}}{\Lambda}\right)=\frac{1}{2 \gamma}\left(\frac{\gamma^{2}}{2 \Lambda}+\frac{\mu^{2}}{3 \Lambda}\right)=\frac{3 \gamma^{2}+2 \mu^{2}}{12 \gamma \Lambda} .
\end{aligned}
$$


From Lemma 3, we derive the expected number of edges intersecting the isosurface and thus the fractal dimension.

\section{Proof of Theorem 1, Statement 1:}

Let $M$ be the number of edges in $E_{\sigma}(\eta)$. By assumption, $M / 8$ equals $\left|E_{\sigma}^{\prime}(\eta)\right| / 8$. Let $\mu$ equal $r \gamma$. The added noise is uniformly distributed in the range $[-\mu, \mu]$. By Lemma 3, the expected value of $I_{e}^{\sigma}(\widetilde{G})$, the number of edges of $\widetilde{G}$ intersecting the isosurface is: $M\left(\frac{32 \mu^{3}+8 \mu \gamma^{2}-\gamma^{3}}{96 \mu^{2} \Lambda}\right)$.

Let $\widetilde{G}^{\prime}$ be the subsampled regular grid of $\widetilde{G}$ with $N / 8$ vertices. Since each subgrid edge covers two edges of grid $\widetilde{G}$, the magnitudes of edge spans of $\widetilde{G}^{\prime}$ vary uniformly from 0 to $2 \gamma$. Thus the expected number of edges of $\widetilde{G}^{\prime}$ intersecting the isosurface is: $(M / 8)\left(\frac{32 \mu^{3}+8 \mu(2 \gamma)^{2}-(2 \gamma)^{3}}{96 \mu^{2} \Lambda}\right)$.

Dividing the number for the full grid by the number for the subsampled grid and replacing $\mu$ by $r \gamma$ gives

$$
\begin{gathered}
8\left(\frac{32 \mu^{3}+8 \mu \gamma^{2}-\gamma^{3}}{32 \mu^{3}+8 \mu(2 \gamma)^{2}-(2 \gamma)^{3}}\right)=8\left(\frac{32(r \gamma)^{3}+8(r \gamma) \gamma^{3}-\gamma^{3}}{32(r \gamma)^{3}+8(r \gamma)(2 \gamma)^{2}-(2 \gamma)^{3}}\right) \\
=8\left(\frac{32 r^{3}+8 r-1}{32 r^{3}+32 r-8}\right)=\frac{32 r^{3}+8 r-1}{32(r / 2)^{3}+8(r / 2)-1} .
\end{gathered}
$$

Thus the expected fractal dimension is: $\log _{2}\left(\frac{32 r^{3}+8 r-1}{32(r / 2)^{3}+8(r / 2)-1}\right)$.

\section{Proof of Theorem 1, Statement 2:}

As in the proof of Statement 2, the number of edges of $\widetilde{G}$ intersecting the isosurface is: $M\left(\frac{32 \mu^{3}+8 \mu \gamma^{2}-\gamma^{3}}{96 \mu^{2} \Lambda}\right)$.

Let $\widetilde{G}^{\prime}$ be the subsampled regular grid of $\widetilde{G}$ with $N / 8$ vertices. The magnitudes of edge spans of $\widetilde{G}^{\prime}$ vary uniformly from 0 to $2 \gamma$. Since $2 \gamma>2 \mu$, Statement 2 of Lemma 3 applies, and the expected number of edges of $\widetilde{G}^{\prime}$ intersecting the isosurface is: $(M / 8)\left(\frac{3(2 \gamma)^{2}+2 \mu^{2}}{12(2 \gamma) \Lambda}\right)$.

Dividing the number for the full grid by the number for the subsampled grid, replacing $\mu$ by $r \gamma$, and taking the logarithm gives Equation 2 .

\section{Proof of Theorem 1, Statement 3:}

By Statement 2 of Lemma 3, the expected number of edges of $\widetilde{G}$ intersecting the isosurface is: $M\left(\frac{3 \gamma^{2}+2 \mu^{2}}{12 \gamma \Lambda}\right)$.

Let $\widetilde{G}^{\prime}$ be the subsampled regular grid of $\widetilde{G}$ with $N / 8$ vertices. The expected number of edges of $\widetilde{G}^{\prime}$ intersecting the isosurface is: $(M / 8)\left(\frac{3(2 \gamma)^{2}+2 \mu^{2}}{12(2 \gamma) \Lambda}\right)$.

Dividing the number for the full grid by the number for the subsampled grid, replacing $\mu$ by $r \gamma$, and taking the logarithm gives Equation 3 .

\section{EXPERIMENTAL RESULTS}

To check the fractal formulas (Equations 1, 2, and 3), we created a point cloud data set representing the Euclidean distance from the origin and added uniform noise in the range $[-\mu, \mu]$ for various values of $\mu \in[0: 4]$. We plotted the resulting fractal edge span dimension and the formula prediction where $r$ equals one. For $\mu \geq 1.0$, Equation 1 applies, for $\mu \in[0.5: 1.0]$, Equation 2 applies, and for $\mu \leq 0.5$, Equation 3 applies. As shown in Figure 7, the prediction matches the experimental data.

In Figure 7, we also plotted the fractal box span dimension. As shown in the figure, the fractal box span dimension is not quite the same as the fractal edge span dimension.

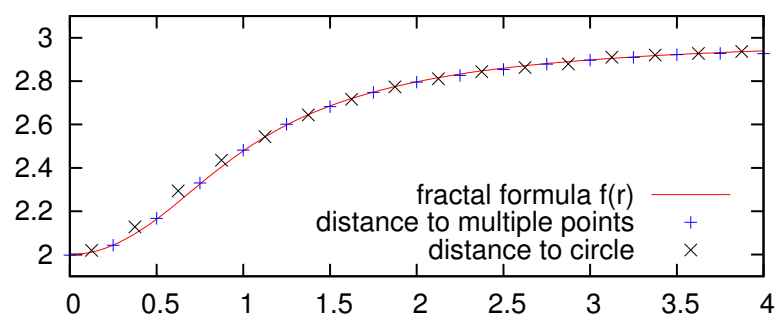

Fig. 8: Plot of fractal edge span dimension versus noise added to a data set representing Euclidean distances to a set of 10 random points. Plot of fractal edge span dimension versus noise added to a data set representing distance to a circle. Plot of fractal formula.

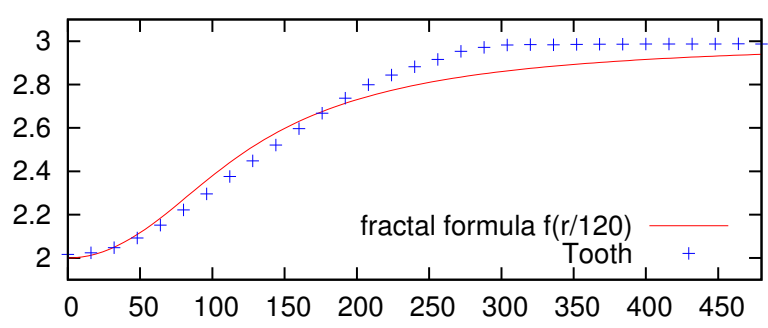

Fig. 9: Plot of fractal dimension (isovalue 650) vs. noise added to the Tooth data set and plot of fractal formula adjusted for the gradient 120 .

As noted in the previous section, sampling the distance function to a finite set of random points also produces a scalar grid satisfying the conditions of Theorem 1. Figure 8 contains the fractal dimensions of the isosurface at the right after noise was added to the data set. The formula prediction again matches the experimental data.

In a third experiment, we created a data set representing the scaled distance to a circle. Isosurfaces in such a data set are tori. The edge spans of this data set are not uniformly distributed and so this data set does not satisfy the conditions of Theorem 1. Nevertheless, the formula does a good job of predicting the fractal dimension. (See Figure 8.)

Finally, we experimented with certain isosurfaces of the benchmark data sets. We looked for isosurfaces where the gradient magnitudes were constant in a neighborhood of the isosurface. If the gradient directions vary uniformly in all directions, then the magnitude of edge spans vary uniformly from 0 to $\gamma$. Examples are Tooth with isovalue 650 and $\gamma$ equal to 120 and Boston Teapot with isovalue 65 and $\gamma$ equal to 25 . Around isovalue $650(+/-50)$, the edge span magnitudes of Tooth vary uniformly from 0 to 120 . Around isovalue 65 (+/-15), the edge span magnitudes of Boston Teapot vary uniformly from 0 to 25 . For each data set and range, the minimum edge scalar values also vary uniformly.

Figures 9 and 10 contain plots of the fractal dimension of the Tooth and Boston Teapot data sets as a function of noise added to those data sets. As can be seen from the figures, the fractal dimension predicted by the formula approximates the experimental data, although not as well as for the synthetic data sets.

\section{Discussion}

The $\Theta\left(N^{0.75}\right)$ isosurface growth estimated in this paper is much smaller than the $\Theta\left(N^{0.82}\right)$ growth estimated in [4] or the $\Theta\left(N^{0.96}\right)$ growth in [18]. The results reported by this paper also did not vary much between the three different categories of data sets, medical, measured and synthetic. This was not the case in [4] or [18]. The 60 data sets used in this paper were a subset of the approximately 80 data sets 


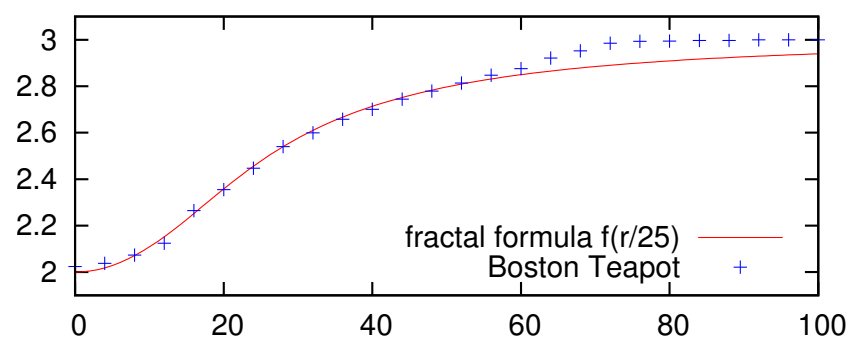

Fig. 10: Plot of fractal dimension (isovalue 65) vs. noise added to the Boston Teapot data set and plot of fractal formula adjusted for the gradient 25 .

used in [18]. We eliminated some apparent duplicates and some synthetic data sets which seemed extremely trivial. We do not believe that the difference in estimates is due to any difference in the set of benchmark data sets.

The authors of [18] suggested that their estimates may have been biased because the benchmark simulated data sets tended to be smaller while the measured and medical data sets were larger. We believe this to be the case. We note that our methodology, which compares each data set with itself, has no such bias. We also think that within a category of data sets, the sample size was too small for the line fitting approach in [18] to provide stable results. In contrast, we measured a standard deviation of 0.16 in the fractal dimension for all the data sets, and of $0.12,0.17$ and 0.22 for the medical, measured and synthetic data sets, respectively.

For many data sets, there is great variance in the topological noise and fractal dimension depending on the isovalue used. Isovalues with small topological noise and fractal dimension tend to be in high gradient regions representing surface boundaries. Isovalues with large topological noise and fractal dimension tend to be in low gradient regions with near constant scalar values. In data sets produced by scanning devices, isosurfaces with isovalues in high gradient regions often represent boundaries between different objects or materials [17] Isosurfaces representing the boundaries between objects are often the most useful for visualization and modeling. The fractal dimension of such isosurfaces may be much lower than the simple average isosurface fractal dimension over all scalar values.

Conversely, isosurfaces in scalar regions with very low gradients often represent a volume consisting of a uniform material. Such regions are not well represented by isosurfaces since they are really three dimensional. (Interval volumes are a better representation.) Thus, regions with high fractal dimension may be the very regions which are least relevant to isosurface construction.

Our formula for the fractal edge span dimension as a function of uniform noise leaves many open questions. First, uniform noise is not the best noise model for most real situations. What is the formula for the fractal edge span dimension as a function of Gaussian noise added to a scalar data set? Second, our formula assumes that the edge span magnitudes are uniformly distributed in the neighborhood of the isovalue. This assumption does not hold for many isosurfaces. What is a more realistic model of the distribution of edge span magnitudes? What is the expected fractal dimension as a function of noise under that model? Thirdly, as shown in Figure 7, the fractal edge span dimension is not quite the same as the fractal box span dimension. What is a formula for the expected box span dimension as a function of noise?

\section{ConClusion}

By subsampling scalar grids, we measured the fractal box span dimension of 60 benchmark data sets. The average fractal box span dimension is 2.26 with an isosurface growth rate of $\Theta\left(N^{0.75}\right)$. We also measured the topological noise in the benchmark data sets and found an average correlation of 0.61 between the topological noise and the fractal box span dimension. Finally, we presented a formula for the fractal edge span dimension as a function of uniform noise added to a data set.

\section{ACKNOWLEDGMENTS}

This research was partially supported by NSF grant CCF-0635008. We thank Michael Meissner and Stefan Roettger for providing public data set repositories at www. volvis.org and www. stereofx.org, respectively. We thank Brian Duffy and Hamish Carr for providing a list of the data sets used in their papers. We also thank Luis Rademacher and Yusu Wang for helpful discussions on the probabilistic analysis.

\section{REFERENCES}

[1] C. Bajaj, V. Pascucci, and D. Schikore. The contour spectrum. In Proc. of IEEE Visualization 1997, pages 167-173, 1997.

[2] P. Bhaniramka, R. Wenger, and R. Crawfis. Isosurface construction in any dimension using convex hulls. IEEE Transactions on Visualization and Computer Graphics, 10(2):130-141, Mar./Apr. 2004.

[3] J. Bloomenthal. An implicit surface polygonizer. In P. Heckbert, editor, Graphics Gems IV, pages 324-349. Academic Press, Boston, MA, 1994.

[4] H. Carr, D. Brian, and D. Barry. On histograms and isosurface statistics. IEEE Transactions on Visualization and Computer Graphics, 12(5):1259-1266, 2006.

[5] H. Carr, J. Snoeyink, and U. Axen. Computing contour trees in all dimensions. Computational Geometry: Theory and Applications, 24:75-94, 2003.

[6] T. K. Dey and J. A. Levine. Delaunay meshing of isosurfaces. Shape Modeling and Applications, International Conference on, pages 241-250, 2007.

[7] K. Falconer. Fractal Geometry. John Wiley and Sons, West Sussex, England, second edition, 2003

[8] S. F. F. Gibson. Constrained elastic surface nets: Generating smooth surfaces from binary segmented data. In MICCAI '98: Proceedings of the First International Conference on Medical Image Computing and Computer-Assisted Intervention, pages 888-898, London, UK, 1998. Springer-Verlag.

[9] T. Itoh and K. Koyamada. Automatic Isosurface Propagation Using an Extrema Graph and Sorted Boundary Cell Lists. IEEE Transactions on Visualization and Computer Graphics, 1(4):319-327, Dec. 1995.

[10] T. Ju, F. Losasso, S. Schaefer, and J. Warren. Dual contouring of hermite data. ACM Trans. Graph., 21(3):339-346, 2002

[11] W. Lorensen and H. Cline. Marching cubes: a high resolution 3d surface construction algorithm. Comput. Graph., 21(4):163-170, 1987.

[12] C. Montani, R. Scateni, and S. Scopigno. A modified look-up table for implicit disambiguation of marching cubes. The Visual Computer, 10(6):353-355, Dec. 1994

[13] T. S. Newman and H. Yi. A survey of the marching cubes algorithm. Computers and Graphics, 30(5):854 - 879, 2006.

[14] G. M. Nielson. On marching cubes. IEEE Transactions on Visualization and Computer Graphics, 9:283-297, 2003.

[15] G. M. Nielson. Dual Marching Cubes. In Proc. of IEEE Visualization 2004, pages 489-496, 2004.

[16] G. M. Nielson and B. Hamann. The asymptotic decider: resolving the ambiguity in Marching Cubes. In VIS '91: Proceedings of the 2nd conference on Visualization '91, pages 83-91. IEEE Computer Society Press, 1991.

[17] V. Pekar, R. Wiemker, and D. Hempel. Fast detection of meaningful isosurfaces for volume data visualization. In VIS '01: Proceedings of the conference on Visualization '01, pages 223-230, Washington, DC, USA, 2001. IEEE Computer Society.

[18] C. E. Scheidegger, J. M. Schreiner, B. Duffy, H. Carr, and C. T. Silva. Revisiting histograms and isosurface statistics. IEEE Transactions on Visualization and Computer Graphics, 14(6):1659-1666, 2008

[19] C. Weigle and D. Banks. Complex-valued contour meshing. In Proceedings of Visualization '96. IEEE Computer Society Press, 1996.

[20] G. Wyvill, C. McPheeters, and B. Wyvill. Data structure for soft objects. The Visual Computer, 2(4):227-234, 1986. 